\title{
Development of an assessment and intervention protocol for postpartum hemorrhage in the mainland of China: an evidence-based method and Delphi consult ${ }^{\dagger}$
}

Original article

Ying Liua, Ya-Jing Fan ${ }^{\mathrm{b}}$, Wei Zhuang ${ }^{\mathrm{c}}$, Qun Huang ${ }^{\mathrm{a}, *}$

${ }^{a}$ Nursing Department, The International Peace Maternity \& Child Health Hospital, Shanghai Jiaotong University School of Medicine, Shanghai 200030, China

${ }^{b}$ Nursing Department, Shanghai 6th People's Hospital Affiliated to Shanghai Jiaotong University School of Medicine, Shanghai 200233, China

cHospital Administration Department, Shanghai First Maternity and Infant Hospital, Shanghai 201204, China

Received: 29 April 2019; Accepted: 2 July 2019; Published: 20 December 2019

Abstract: Objective: Postpartum hemorrhage $(\mathrm{PPH})$ is a leading cause of maternal death. Although guidelines have been updated, those with detailed protocols are limited for nursing practice. This study aims at establishing an early assessment and intervention protocol as a toolkit for PPH for midwives and obstetrical nurses.

Methods: Employing the evidence-based method, a systematic Internet search of guidelines was conducted and appraisal of literatures was conducted with AGREE system and Oxman-Guyatt Overview Quality Assessment Questionnaire (OQAQ), according to which a protocol draft was therefore developed. Then, a two-round modified Delphi method was utilized to reach a consensus of the protocol built on best practices. Selection criteria for each intervention measure included consensus level with a threshold of $70 \%$, mean of importance $(M)>3.5$, and coefficient of variation $(C V)<0.25$. Reliability of experts' opinion was calculated by positive coefficient and authoritative coefficient. Items without consistency were enlisted in the second-round consult. When all items met the selection criteria, the protocol would be finally formulated.

Results: A 122-measure protocol was established, including prevention, assessment, and intervention of PPH. With a panel of 14 experts participated in the consult, the positivity coefficient was 0.93 and 1.00 for two rounds, respectively, and the authority coefficient was 0.88 . After a two-round consult and revision of the draft, the final program was formulated, containing 5 first-level indexes and 14 second-level indexes with a total item of 120.

Conclusions: The PPH protocol, based on high-quality evidences, was formulated with a two-round Delphi method, which can provide insight for midwives and obstetrical nurses to effectively deal with PPH.

Keywords: postpartum hemorrhage $\bullet$ assessment $\bullet$ intervention $\bullet$ protocol $\bullet$ evidence-based method $\bullet$ modified Delphi technique (c) Shanxi Medical Periodical Press.

${ }^{\dagger}$ This project was supported by Nursing Research Program of Shanghai Nursing Association (No. 2016SD-BO3).

How to cite this article: Liu Y, Fan YJ, Zhuang W, Huang Q. The development of an assessment and intervention protocol for postpartum hemorrhage in the mainland of China: an evidence-based method and Delphi consult. Front Nurs. 2019; 4: 285-292. 


\section{Introduction}

With slow but steady progressions, China has met its millennium development goals (MDG) target for reducing the under-five maternal mortality rate (MMR) in 2009. ${ }^{1}$ Yet, a comparatively large gap still exists in MMR between China and developed countries. Postpartum hemorrhage $(\mathrm{PPH})$, constantly known as the leading cause of maternal death, was high $(26.3 \%)$ as presented in the 2015 China Health Statistics Yearbook. ${ }^{2}$ There was dramatic regional differences - urban areas were reported $21.2 \%$, whereas rural areas $28.3 \%$, giving clues to a strike disparity of medical level across the country.

In 2016, the "two-child policy" in the mainland of China has comprehensively commenced, which is estimated to bring about a "Baby Boom" around 2018 and a peak in 2026. ${ }^{3}$ However, advanced maternal age, increasing incidence of maternal complications, multiple pregnancies caused by artificial reproduction technology, as well as cicatricial uterus bring greater challenges for obstetrical health professionals. Moreover, limited midwives available adds to enormous threats to the safety of both maternities and newborns. According to a survey on midwife staffing in 31 different provinces in China, the numbers of midwives per 1,000 people in East, Middle, and West China are $0.040,0.028$, and 0.033 , respectively. ${ }^{4}$ Among them, $43.4 \%$ has a diplomat of associate degree and $41.9 \%$ graduated from specialized secondary schools with a technical degree. Meanwhile, midwives transferred from nurses made up a considerable proportion, who might not be competent enough without systematic midwifery education. In fact, China did not launch a full-time 4-year bachelor's program of midwifery until 2014 , and there will be a long way to go before advanced nurse midwives with master or doctoral degrees are available. Under this circumstance, essential knowledge and skills are critical for midwives to guarantee a promising quality of maternal care.

Midwives participated in midwife training programs demonstrated knowledge improvement and better competencies and performances when dealing with $\mathrm{PPH}^{5,6}$ As death caused by PPH can be somewhat preventable and controllable, it is imperative to make efforts to reduce its incidence. In 2014, the Obstetrics and Gynecology Branch of Chinese Medical Association updated previous $\mathrm{PPH}$ guideline to provide instructions for obstetrical professionals. ${ }^{7}$ Nevertheless, with a primary focus on medical treatment, this guideline played a limited role for midwives and nurses. In line with international guidelines, the scope of its clinical practice has been largely restrained to advanced practitioners, whereas little can be done by domestic midwives according to it. A systematic assessment and intervention protocol is in urgent need to standardize clinical routine improve the ability of midwives to manage PPH. Consequently, we constructed a protocol to customize domestic conditions, on the basis of evidence-based practices and quality appraisal of recommendations. This study aimed at testifying the practicality and validity of the protocol with a two-round modified Delphi consult; thus, it can be applied in local institutions.

\section{Methods}

\subsection{Design}

Delphi Technique is "a method used to obtain the most reliable consensus of a group of experts by a series of intensive questionnaires interspersed with controlled feedback." ${ }^{8}$ As a modified version, the draft was grounded on existing evidence-based guidelines, systematic reviews, and meta-analyses.

\subsection{Generation of item list}

Before the formulation of the protocol draft, the research group went through a systematic search of electronic databases and professional websites for worldwide $\mathrm{PPH}$ guidelines and meta-analyses, including Cochrane Library, Joanna Briggs Institute Library, National Guideline Clearinghouse (NGC), Scottish Intercollegiate Guidelines Network (SIGN), the National Institute for Health and Care Excellence (NICE), Registered Nurses' Association of Ontario (RNAO), Pubmed, Medline, Embase, CINAHL, CBM (SinoMed), China National Knowledge Infrastructure (CKNI), Wanfang, WHO, ACOG, SOGC, and RCOG. The Appraisal of Guidelines for Research \& Evaluation ${ }^{9}$ and Oxman-Guyatt Overview Quality Assessment Questionnaire ${ }^{10}$ were adopted for a rigorous quality appraisal, after which reports rated as "low" or "not recommended" were excluded from the item list. Following the extraction of high-quality recommendations and a group discussion, a first-level index of five dimensions was formed, containing prevention strategies, assessment strategies, intervention strategies, facility and health professional support, and $\mathrm{PPH}$ patient education. In all, 14 aspects of the second-level index are as follows: prenatal risk scoring, active management of the third stage of labor (AMTSL), Airway-Breathing-Circulation (ABC) assessment, assessment of blood loss, Tone-Trauma-TissueThrombin (4T) assessment, conscious assessment, mild PPH (blood loss 500-1000 mL) interventions, moderate PPH (blood loss 1000-2000 mL) interventions, severe $\mathrm{PPH}$ (blood loss above $2000 \mathrm{~mL}$ ) interventions, nursing care after rescue, facility workflow, midwife and nurse training, health education for maternity and family, and follow-up. A total of 122 items were expanded. Given that 
comprehensive assessment indicators and procedures were rarely mentioned in those recommendations, a prospective study ${ }^{11}$ of 24 -hour postpartum observation was conducted before the first round to supplement assessment strategies.

\subsection{Selection of experts}

Experts are those who have more knowledge of the topic under investigation than most people. ${ }^{12}$ The expert panel was carefully chosen by the study group with the following standards: (a) engaged in obstetrical clinic or clinical management; (b) master's degree or above for obstetricians while bachelor's degree or above for midwives and nurses; (c). mid-level title or above; (d) at least 8 years of clinical experiences; and (e) abundant professional knowledge and rigid academic custom, as well as inclination for participating in this consult. A total sample size was made 15 as recommended by Skulmoski ${ }^{13}$ that a smaller sample size, such as $10-15$ participants, may be sufficient if the sample is homogeneous.

\subsection{Procedure}

Prior to the consult process, all panel members were offered explicit information about the study. Questionnaires were sent to experts with information consent. For each round, 2 weeks was given to the panelists, after which papers were collected by the researcher (Y. L.) in person. Furthermore, each expert was provided with remunerations before the end of the second consult round. Ethnic approval was not applicable.

\subsection{Data analysis}

SPSS 21.0 software was applied for data analysis. Both the reliability of the expert panels and agreement of listed items were analyzed. The former included demographic descriptions, positive coefficient, and authoritative coefficient. The pre-determined coefficients were set 0.7. Parameters involved were calculated for reliability as below.

Positive coefficient $\left(C_{p}\right)$ is characterized by the ratio of those experts actually participated $\left(M_{p}\right)$ to those selected $(M)$ (computation formula: $C_{p}=M_{p} / M$ ). Another indicator, authoritative coefficient $\left(C_{a}\right)$, is correlated to two variables, namely basis of judgment $\left(C_{j}\right)$ and familiarity of items $\left(C_{\mathrm{f}}\right)$. This can be calculated with the following process: (1) first, each expert rated the influence levels of their judgment as "high, moderate or low", regarding theoretical analysis, empirical evidence, peer communication, and intuition. The quantification of each level is displayed in Table 1. $C_{j}$ can be calculated with the

\begin{tabular}{lccc}
\hline \multirow{2}{*}{ Basis of judgment } & \multicolumn{3}{c}{ Influence level } \\
\cline { 2 - 4 } & High & Moderate & Low \\
\hline \hline Theoretical analysis & 0.3 & 0.2 & 0.1 \\
Empirical evidence & 0.5 & 0.4 & 0.3 \\
Peer communication & 0.1 & 0.1 & 0.1 \\
Intuition & 0.1 & 0.1 & 0.1 \\
Total & 1.0 & 0.8 & 0.6 \\
\hline
\end{tabular}

Table 1. Basis of judgment and quantification of influence levels.

formula $C_{j}=\Sigma M_{j} W_{j} / M_{p}$, whereas $M_{j}$ stands for the number of experts who chose a certain level of one basis, and $W_{\mathrm{j}}$ for weight of quantifications. For each basis, $C_{j}$ is counted separately. (2) Then, experts self-evaluated the overall familiarity of all questions surveyed for levels of "very familiar, familiar, modest, unfamiliar, and very unfamiliar," with the weight coefficients of $1.0,0.8$, $0.6,0.4$, and 0.2 , respectively. The parameter can be reached with formula $C_{f}=\Sigma M_{f} W_{f} / M_{p}$. (3) Finally, authoritative coefficient $\left(C_{\mathrm{a}}\right)$ can be determined as follows: $C_{\mathrm{a}}=\left(C_{\mathrm{j}}+C_{\mathrm{f}}\right) / 2$.

To screen items from the draft, each item was evaluated for importance using a 5-point Likert scale (5 = strongly agree, 4 = agree, 3 = neutral, 2 = disagree, and $1=$ strongly disagree). The criteria for item selection were level of consensus with a threshold of $70 \%$, $M>3.5$ and $\mathrm{CV}<0.25$. This consult ended after two rounds since consensus was achieved for the majority. Particularly, only items without consistency in first round were included in the next round survey for the sake of a high response rate.

\section{Results}

\subsection{Demographic of the expert panel}

In total, 14 experts engaged in the consult, and one dropped for the reason of being busy. As shown in Table 2, the average age of the panelist was $44.07 \pm 8.23$, ranging from 33 to 57 , whereas working experience (in years) $22.71 \pm 9.27$, ranging from 10 to 38 . Among these experts, four were majoring in medicine while the rest involved with nursing or midwifery. Half owned a degree of master or doctoral and the title of associate professor or professor, or that equals. The expert panel was experienced and well representative.

\subsection{Reliability of the expert panel}

Positive coefficient in the first round was 0.93 (i.e., $C_{p}=M_{p} / M=14 / 15 \approx 0.93$ ), and the second round 1.00 . 
Besides, 12 experts offered advices in the feedback of the first round.

Scores of judging bases are shown in Table 3. Judgment coefficients of theoretical analysis, empirical evidence, peer communication, and intuition were $0.29,0.35,0.10$, and 0.10 , respectively. Therefore, the total of judgment coefficient was 0.84 . Normally, a value of 1.0 suggests a greater influence of expert's judgment, while 0.8 moderate and 0.6 mild. The level of familiarity was 0.91 in weighed average $\left(C_{\mathrm{f}}=\Sigma M_{\mathrm{f}} W_{\mathrm{f}} / M_{\mathrm{p}}=(8 \times 1.0+6 \times 0.8+0 \times 0.6+0 \times\right.$ $0.4+0 \times 0.2) / 14)$. The authoritative coefficient for this expert panel was approximately 0.88 , which indicated a comparatively fair reliability.

\begin{tabular}{|c|c|c|}
\hline Demographics & Number of experts & Percentage (\%) \\
\hline \multicolumn{3}{|l|}{ Gender } \\
\hline Male & 1 & 7.14 \\
\hline Female & 13 & 92.86 \\
\hline \multicolumn{3}{|l|}{ Age (years) } \\
\hline 30-39 & 6 & 42.86 \\
\hline $40-49$ & 3 & 21.43 \\
\hline$\geq 50$ & 5 & 35.71 \\
\hline \multicolumn{3}{|l|}{ Title } \\
\hline Lecturer* & 7 & 50.00 \\
\hline Associate professor* & 3 & 21.43 \\
\hline Professor* & 4 & 28.57 \\
\hline \multicolumn{3}{|l|}{ Academic degree } \\
\hline Bachelor & 9 & 64.29 \\
\hline Master & 4 & 28.57 \\
\hline $\mathrm{PhD}$ & 1 & 7.14 \\
\hline \multicolumn{3}{|l|}{ Profession } \\
\hline Medicine & 4 & 28.57 \\
\hline Nursing & 10 & 71.43 \\
\hline \multicolumn{3}{|l|}{ Working experience (years) } \\
\hline $10-19$ & 6 & 42.86 \\
\hline $20-29$ & 4 & 28.57 \\
\hline$\geq 30$ & 4 & 28.57 \\
\hline
\end{tabular}

Note: *means "is" or "equals to".

Table 2. Demographic characters of the expert panel $(n=14)$.

\begin{tabular}{lccc}
\hline \multirow{2}{*}{ Basis of judgment } & \multicolumn{3}{c}{ Influence level } \\
\cline { 2 - 4 } & High & Moderate & Low \\
\hline \hline Theoretical analysis & 13 & 1 & 0 \\
Empirical evidence & 13 & 1 & 0 \\
Peer communication & 7 & 6 & 1 \\
Intuition & 4 & 3 & 7 \\
\hline
\end{tabular}

Table 3. Self-evaluation of the basis of judgment.

\subsection{Level of agreement}

\subsubsection{Round 1}

Of the 122 items contained, level of consensus ranged from $42.86 \%$ to $100 \%$, with 19 below $70 \%$ for another round of consult; the average scores of significance varied from 3.86 to 5.00 , whereas $\mathrm{CV}$ differed from 0.00 to 0.35 , with five items over 0.25 . Furthermore, since 11 items were raised to be modified, research group went them over for review and included the amendments in the next round as well. Items included for the second round consult are shown in Table 4.

\subsubsection{Round 2}

The second round embraced 28 items, most of which had met consensus with level of agreement between $78.57 \%$ and $100.00 \%$, mean of importance score 4.21-4.93, and CV 0.06-0.23, except for three items that were eliminated due to inconformity. Reasons for exclusion were demonstrated as below:

a. The item "percentage of blood loss needs to be calculated" was removed for only four experts chose a score of 5 or 4 . Given that there has not been domestic report on this method for estimating of blood loss, which to some extent reflected its rare application in clinical practice, research group agreed to delete it.

b. Though previously proposed by an expert that "controlled cord traction (CCT) is introduced if there is no sign of placenta separation for 15 min," the suggestion was declined in the second round. Besides, 10 experts considered a 15-min observation span to be unsound, and that compliance with guidelines was both reasonable and practical. Consequently, the interval was changed back into $30 \mathrm{~min}$.

c. In intervening PPH, the strategy of aortic artery compression manifested a consensus level of $28.57 \%$ and mean of importance 3.50 , analogous to the first round. Although this method has been reported effective in previous studies, ${ }^{14}$ it is uncommonly implemented in China. With only one report found in Chinese database and none else in Asian countries internationally, its safety and efficiency remain ambiguous among Asian population. To comply with domestic practice, this method was canceled.

Since the majority of these items reached consensus, the final content of the program was set and consult rounds ended. The warning assessment and intervention program ultimately formulated contained 5 first-level 


\begin{tabular}{|c|c|c|c|c|c|c|}
\hline \multirow{2}{*}{ First level } & \multirow{2}{*}{ Second level } & \multirow{2}{*}{ Three level } & \multirow{2}{*}{ Agreement (\%) } & \multicolumn{3}{|c|}{ Scoring of importance } \\
\hline & & & & $\mathrm{M}$ & SD & CV \\
\hline \multirow{2}{*}{$\begin{array}{l}\text { I-1 Prevention } \\
\text { Strategies }\end{array}$} & II-2 AMTSL & Late cord clamping & 50.00 & 4.07 & 1.21 & 0.30 \\
\hline & & ССТ & 42.86 & 4.00 & 1.11 & 0.28 \\
\hline \multirow{3}{*}{$\begin{array}{l}\text { I-2 Assessment } \\
\text { Strategies }\end{array}$} & II-3 ABC Assessment & Airway assessment & 50.00 & 4.21 & 0.89 & 0.21 \\
\hline & & Breathing assessment & 50.00 & 4.29 & 0.83 & 0.19 \\
\hline & II-4 Blood loss assessment & $\begin{array}{l}\text { Assessing blood amount: calculate the } \\
\text { percentage of blood loss when over } 500 \mathrm{~mL}\end{array}$ & 42.86 & 4.00 & 1.11 & 0.28 \\
\hline \multirow[t]{19}{*}{$\begin{array}{l}\text { I-3 Intervention } \\
\text { Strategies }\end{array}$} & $\begin{array}{l}\text { II-7 Mild PPH (blood loss } \\
500-1000 \mathrm{~mL} \text { ) }\end{array}$ & $\begin{array}{l}\text { Fluid management: choose \#16 or larger } \\
\text { needles }\end{array}$ & 92.86 & 4.93 & 0.27 & 0.06 \\
\hline & & $\begin{array}{l}\text { Airway management: assess the breath sound } \\
\text { and apply oxygen therapy for } 6-10 \mathrm{~L} / \mathrm{min}\end{array}$ & 64.29 & 4.57 & 0.65 & 0.14 \\
\hline & & $\begin{array}{l}\text { Continuous assessment: electrocardiogram } \\
\text { monitor is applied }\end{array}$ & 85.71 & 4.86 & 0.36 & 0.07 \\
\hline & & Uterine atony: uterine massage & 64.29 & 4.50 & 0.76 & 0.17 \\
\hline & & $\begin{array}{l}\text { Birth canal injury: check for hematoma, } \\
\text { cut it for drainage, suture or compress for } \\
\text { hemostasis }\end{array}$ & 71.43 & 4.71 & 0.47 & 0.10 \\
\hline & & $\begin{array}{l}\text { Observe for signs of placenta abruption, } \\
\text { and palpate the uterus fundus to assess } \\
\text { contraction }\end{array}$ & 42.86 & 4.07 & 1.14 & 0.28 \\
\hline & & $\begin{array}{l}\text { CCT is performed for vaginal births once the } \\
\text { placenta has been retaining for } 30 \mathrm{~min}\end{array}$ & 64.29 & 4.50 & 0.76 & 0.17 \\
\hline & & Apply ultrasonography if available & 50.00 & 4.36 & 0.75 & 0.17 \\
\hline & $\begin{array}{l}\text { II-8 Moderate PPH (blood } \\
\text { loss 1000-2000 mL) }\end{array}$ & $\begin{array}{l}\text { Airway management: assess the breath sound } \\
\text { and apply oxygen therapy for } 10-15 \mathrm{~L} / \mathrm{min}\end{array}$ & 71.43 & 4.64 & 0.63 & 0.14 \\
\hline & & Establish the second venous access & 92.86 & 4.93 & 0.27 & 0.06 \\
\hline & & $\begin{array}{l}\text { Adjust the infusion rate to the maximum and } \\
\text { apply pressure to the fluid bag if necessary }\end{array}$ & 85.71 & 4.86 & 0.36 & 0.07 \\
\hline & & $\begin{array}{l}\text { Body position management: change into } \\
\text { supine or Trendelenburg's position }\end{array}$ & 64.29 & 4.64 & 0.50 & 0.11 \\
\hline & & $\begin{array}{l}\text { Temperature management: use warm fluid or } \\
\text { heating device, and keep fluid temperature } \\
\text { around } 40\end{array}$ & 64.29 & 4.64 & 0.50 & 0.11 \\
\hline & & $\begin{array}{l}\text { Vital signs assessment: assess temperature } \\
\text { every } 15 \mathrm{~min}\end{array}$ & 42.86 & 3.86 & 1.35 & 0.35 \\
\hline & & $\begin{array}{l}\text { Urine assessment: every } 15 \text { min after } \\
\text { catheterization }\end{array}$ & 85.71 & 4.64 & 0.93 & 0.20 \\
\hline & & $\begin{array}{l}\text { Uterine atony: apply hemostatic drugs if } \\
\text { uterotonics fails or trauma exists }\end{array}$ & 42.86 & 4.14 & 0.77 & 0.19 \\
\hline & & Uterine atony: abdominal aortic compression & 42.86 & 4.29 & 0.73 & 0.17 \\
\hline & $\begin{array}{l}\text { II- } 10 \text { Nursing care after } \\
\text { rescue }\end{array}$ & $\begin{array}{l}\text { Hemodynamic changes: observe for } 2 \text { hours in } \\
\text { the delivery room before transferring }\end{array}$ & 92.86 & 4.93 & 0.27 & 0.06 \\
\hline & & $\begin{array}{l}\text { Hemodynamic changes: observe for vital } \\
\text { signs, uterine contraction and loss of blood } \\
\text { every } 4 \text { hours in the ward for the first } 24 \text { hours }\end{array}$ & 92.86 & 4.93 & 0.27 & 0.06 \\
\hline \multirow[t]{4}{*}{$\begin{array}{l}\text { I-5 Health Education } \\
\text { after PPH }\end{array}$} & $\begin{array}{l}\text { II-13 Health education for } \\
\text { puerpera and family }\end{array}$ & Prevention of complications: anemia & 64.29 & 4.64 & 0.50 & 0.11 \\
\hline & & $\begin{array}{l}\text { Prevention of complications: Sheehan } \\
\text { syndrome }\end{array}$ & 50.00 & 4.43 & 0.65 & 0.15 \\
\hline & & Breast feeding instructions & 64.29 & 4.57 & 0.65 & 0.14 \\
\hline & & Psychological instructions & 64.29 & 4.50 & 0.76 & 0.17 \\
\hline
\end{tabular}

Note: AMTSL: Active Management of the Third Stage of Labor; ABC Assessment: Airway-Breathing-Circulation Assessment; CCT: Controlled Cord Traction; PPH: postpartum hemorrhage.

Table 4. Items included for the second-round consult. 
indexes, 14 second-level indexes of 120 specific intervening measures.

\section{Discussion}

Whereas the content included in conventional Delphi technique was mainly qualitative interviews or expert meeting, ${ }^{12}$ this modified version considered an evidence-based review approach to provide scientific instructions for clinical midwives. In line with present authoritative guidelines, ${ }^{1,15,16}$ details of prevention and intervention management were integrated as a solid scientific foundation of the program. To improve the comprehensive ability to manage $\mathrm{PPH}$ and holistic nursing, interventions that were rarely covered in guidelines were supplemented to this program from other forms of high-quality evidences, such as meta-analyses, which also explained the necessity of the consult.

Raised as preventative measurements, AMTSL is what extant guidelines consider to be effective. Notably, a screening tool for high-risk population was introduced to the preventative strategies, as has been reported to lower the chances of PPH incidence since some of them can be controlled. Due to different sensitivities of existing tools, we therefore conducted a meta-analysis ${ }^{17}$ to compare the accuracy of each one and added into the program those with better prediction. According to agreement level, the number of experts who rated warning screening as important is even more than that of AMTSL. Professionals can be timely alerted by these warning scores. In light of limited competency of PPH treatment for hospital in less developed areas, it also serves to timely transfer high-risk population into tertiary facilities, thereby reducing the morbidity of severe $\mathrm{PPH}$.

Thorough assessment indicators were elucidated in the program, combining international practice standard $^{18}$ and the results of a 24 -hour prospective study with repeated-measures analysis. Pulses, blood pressure, and shock index, in resonant with a previous meta-analysis, were employed as primary indicators, while others as secondary, such as urine, $\mathrm{SaO}_{2}$, uterine fundus, and so forth. ${ }^{19}$ Provided that assessment strategies were not emphasized in detail in the national guideline, we joint the concepts of "ABC and 4T" assessment frequently used in emergency management by western facilities. The panel's consensus was similar to the expert opinion where "ABC and 4T" were mentioned, except that the item of airway examination got a $50 \%$ approval rate in the first round. As airway failure rarely occurs in vaginal birth, ${ }^{20}$ some experts showed a tendency toward exclusion.

Though seldomly recommended in most guidelines, $\mathrm{PPH}$ training for health professionals has been proved to be effective, not only for improving the level of relevant knowledge but also promoting the performance of $\mathrm{PPH}$ simulations. ${ }^{5}$ That is why training took such an important role in this protocol. One proposal was dismissed in the first round feedback that standardized visual estimation training should be canceled for its inaccuracy. ${ }^{21}$ While it is true that objective measurements are favored, ${ }^{22}$ we should not deny that visual estimation is widely used as a rapid way to suspect $\mathrm{PPH}$ and activate the patient assessment, ${ }^{23}$ especially when obstetrical workload is so prominent for such an enormous population. That is why we are currently processing a systematic training protocol, and designing the evaluation method.

Continuous care for anemia and potential postpartum depression was barely acceded in the part of health education. This goes consistent with the opinion of Holm et al. ${ }^{24}$ that clinical outcomes are constantly ignored by researchers who paid more attention to superior parameters of $\mathrm{Hb}$ and iron in those manifested with fatigue and deficient cognitive functions and mood disturbances. The qualified levels of agreement might be ascribed to limited abilities of domestic healthcare providers (HCP) to recognize postpartum depression ${ }^{25}$ and other psychological disorders. ${ }^{26}$ This also warrants further investigation on relevant fields, not only to improve professional competence to identify mental problem but also to substantially increase the quality of care after $\mathrm{PPH}$.

\section{Conclusions}

On the basis of evidence-based method, the Assessment and Intervention Protocol for PPH was formulated under a two-round modified Delphi consult, with 120 intervention measures covering 14 aspects within 5 first-level indexes of prevention strategies, assessment strategies, intervention strategies, facility and health professional support, and $\mathrm{PPH}$ patient education. Oriented as a directive toolkit for clinical practice of $\mathrm{PPH}$, clinical application is to be implemented for a sounder foundation of its practicality. Through the training course of this program, midwives and nurses are expected to equip with better capacity to deal with PPH as early as possible in an evidence-based way.

\section{Acknowledgment}

This project was supported by Nursing Research Program of Shanghai Nursing Association (No. 2016SD-B03).

\section{Ethics approval}

Ethical issues are not involved in this paper.

\section{Conflicts of interest}

All contributing authors declare no conflicts of interest. 


\section{References}

1. World Health Organization. WHO Recommendations for the Prevention and Treatment of Postpartum Haemorrhage; 2012. http://www.who.int/reproductivehealth/publications/maternal_perinatal_ health/9789241548502/en/. Accessed February 17, 2018.

2. National Health and Family Planning Commission of the People's Republic of China. 2015 China Statistical Yearbook of Health and Family Planning. Beijing: Pecking Union Medical College Press; 2016.

3. Yao J, Zhu X, Lu H. Assessing the midwifery workforce demand: utilising birthrate plus in China. Midwifery. 2016;42:61-66.

4. Li DD, Luo SS, An L. Study on the current situation of midwifery personnel in China. Matern Child Health Care China. 2014;29:993-996 (in Chinese).

5. Kato C, Kataoka Y. Simulation training program for midwives to manage postpartum hemorrhage: a randomized controlled trial. Nurse Educ Today. 2017;51:88-95.

6. Chen P, Gao Q, Chen M, et al. The accuracy of visual assessment for estimating blood loss of vaginal delivery after training: a prospective study. Chin J Pract Obstet Gynecol. 2014;30:851-853 (in Chinese).

7. Liu XH. Prevention and treatment of postpartum hemorrhage. Chin J Obstet Gynecol. 2014;9: 641-646 (in Chinese).

8. Dalkey N, Helmer O. Delphi technique: characteristics and sequence model to the use of experts. Manage Sci. 1963;9:458-467.

9. AGREE Next Steps Consortium. The AGREE II Instrument [Electronic version]; 2009. http://www. agreetrust.org. Accessed February 17, 2018.

10. Oxman $A D$, Guyatt $G H$. Validation of an index of the quality or review articles. $J$ Clin Epidemiol. 1991;44:1271-1278.

11. Liu $Y$, Huang $Q, Y e W Q$. The assessment and observation of postpartum hemorrhage in vaginal delivery. Chin J Mod Nurs. 2017;23:23-27 (in Chinese).

12. Keeney S, Hasson F, McKenna H. The Delphi Technique in Nursing and Health Research. United Kingdom: Wiley-Blackwell; 2011:96-104.

13. Skulmoski GJ, Hartman FT, Krahn J. The Delphi method for graduate research. $J$ Inform Technol Educ. 2007;6:1-21.

14. Soltan MH, Imam H, Zahran KA, et al. Assessing changes in flow velocimetry and clinical outcome following use of an external aortic compression device in women with postpartum hemorrhage. Int $J$ Gynecol Obstet. 2010;110:257-261.

15. Guideline Summary NGC- 005702. Postpartum hemorrhage; 2013. https://www.guideline.gov/ summaries/summary/10922. Accessed February 17, 2018.

16. Mavrides E, Allard S, Chandraharan E, et al. Prevention and management of postpartum haemorrhage. RCOG Green-top Guideline No. 52; 2016. https://www.rcog.org.uk/en/guidelines-researchservices/guidelines/gtg52/. Accessed February 17, 2018.

17. Liu $Y$, Huang $Q$, Wei $X M$, Ye WQ. Predictive effects of postpartum hemorrhage warning scores on postpartum hemorrhage: a meta-analysis. Nurs J Chin PLA. 2017;34:13-17 (in Chinese).

18. Queensland Maternity and Neonatal Clinical Guidelines Program. Primary Postpartum Hemorrhage. Queensland Maternity and Neonatal Clinical Guidelines; 2012. http://creativecommons.org/licenses/bync-nd/3.0/au/deed.en. Accessed February 17, 2018.

19. Pacagnella RC, Souza JP, Durocher J, et al. A systematic review of the relationship between blood loss and clinical signs. PLOS ONE. 2013;8:575-594.

20. Cooper GM, McClure JH. Anaesthesia chapter from Saving mothers' lives; reviewing maternal deaths to make pregnancy safer. $\mathrm{Br} J$ Anaesth. 2008;100:17-22.

21. Rothermel LD, Lipman JM. Estimation of blood loss is inaccurate and unreliable. Surgery. 2016;160:946-953.

22. Lertbunnaphong $T$, Lapthanapat $N$, Leetheeragul J, Hakularb P, Ownon A. Postpartum blood loss: visual estimation versus objective quantification with a novel birthing drape. Singapore Med J. 2016;57:325-328.

23. Natrella M, Di Naro E, Loverro M, et al. The more you lose the more you miss: accuracy of postpartum blood loss visual estimation. A systematic review of the literature. J Matern Fetal Neonatal Med. 2017;12:1-13.

24. Holm C, Thomsen LL, Norgaard A, et al. Singledose intravenous iron infusion versus red blood cell transfusion for the treatment of severe postpartum anemia: a randomized controlled pilot study. Vox Sang. 2017;112:122-131.

25. Zhong BL, Cui J, Chen HH, et al. Investigation of postpartum depression among medical staff from 21 maternal and child care centers in Wuhan: a cross-sectional survey. Chin J Obstet Gynecol Pediatr (Electron Ed). 2011;7: 13-16 (in Chinese).

26. Hu HY, Mei F, Lin XM, Li J, Zhou DH. Recognition of postpartum psycho-behavior problems among medical staff from maternity and child medical institutions in Guangzhou. Chin J Health Psychol. 2012;20:525-528 (in Chinese). 